Provided for non-commercial research and education use. Not for reproduction, distribution or commercial use.

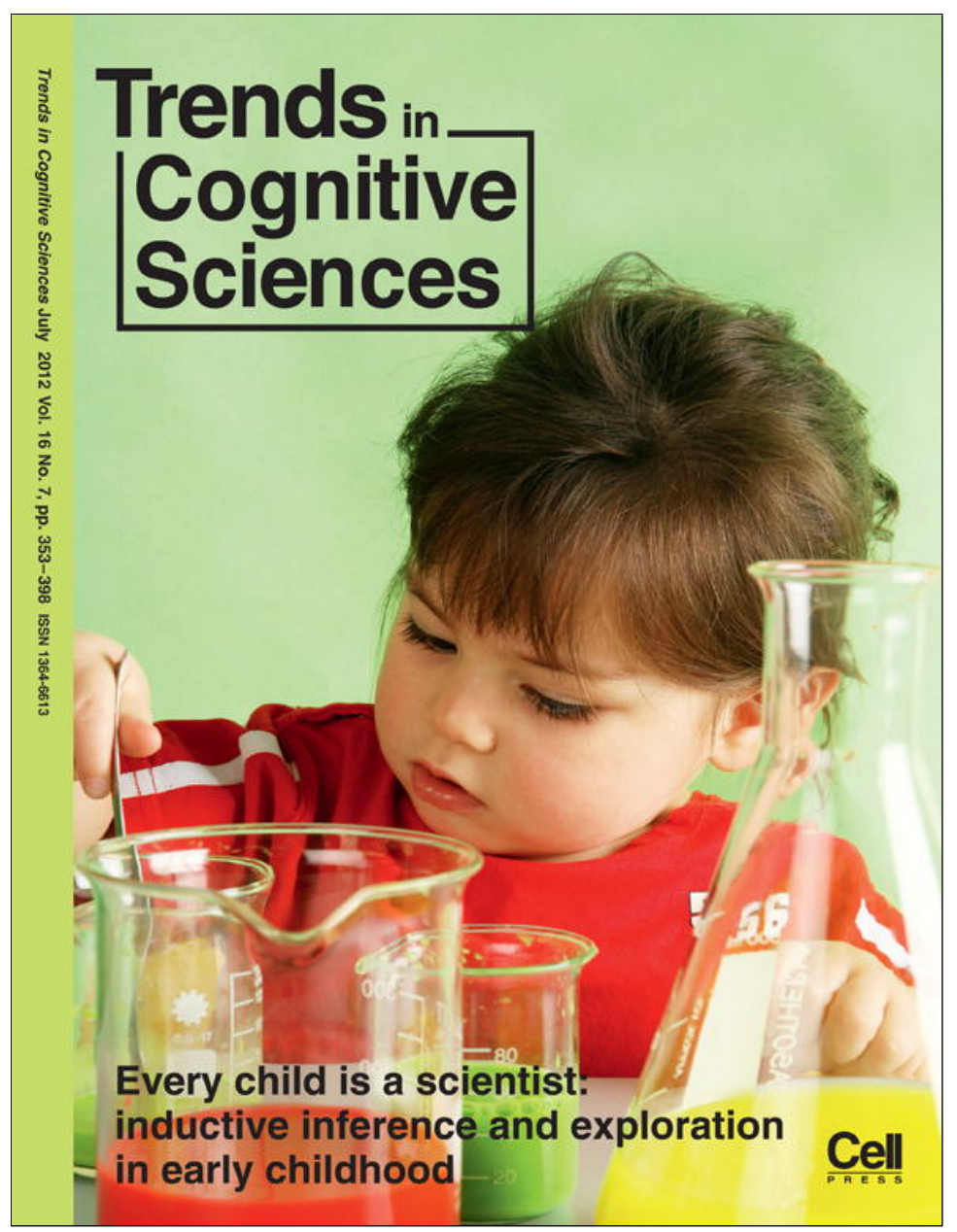

This article appeared in a journal published by Elsevier. The attached copy is furnished to the author for internal non-commercial research and education use, including for instruction at the authors institution and sharing with colleagues.

Other uses, including reproduction and distribution, or selling or licensing copies, or posting to personal, institutional or third party websites are prohibited.

In most cases authors are permitted to post their version of the article (e.g. in Word or Tex form) to their personal website or institutional repository. Authors requiring further information regarding Elsevier's archiving and manuscript policies are encouraged to visit:

http://www.elsevier.com/copyright 


\title{
Affect-biased attention as emotion regulation
}

\author{
Rebecca M. Todd ${ }^{1}$, William A. Cunningham ${ }^{1,2}$, Adam K. Anderson ${ }^{1}$ and \\ Evan Thompson ${ }^{3}$
}

\author{
${ }^{1}$ Department of Psychology, University of Toronto, 100 St George Street, Toronto, ON M5S 3GM, Canada \\ ${ }^{2}$ Department of Psychology, Ohio State University, 1827 Neil Avenue, Columbus, OH 43210, USA \\ ${ }^{3}$ Department of Philosophy, University of Toronto, 170 St George Street, Toronto, ON M5R 2M8, Canada
}

\begin{abstract}
The affective biasing of attention is not typically considered to be a form of emotion regulation. In this article, we argue that 'affect-biased attention' - the predisposition to attend to certain categories of affectively salient stimuli over others - provides an important component of emotion regulation. Affect-biased attention regulates subsequent emotional responses by tuning one's filters for initial attention and subsequent processing. By reviewing parallel research in the fields of emotion regulation and affect-biased attention, as well as clinical and developmental research on individual differences in attentional biases, we provide convergent evidence that habitual affective filtering processes, tuned and re-tuned over development and situation, modulate emotional responses to the world. Moreover, they do so in a manner that is proactive rather than reactive.
\end{abstract}

Affect-biased attention is a form of emotion regulation Emotion regulation, defined as 'all of the conscious and nonconscious strategies we use to increase, maintain, or decrease one or more components of an emotional response' [1], encompasses a wide array of strategies, ranging from implicit to explicit and reactive to effortful (see Glossary), that can be applied in anticipation of or in response to an emotional stressor [2,3]. Yet, despite the range of processes studied under the rubric of emotion regulation, including a number of attentional processes, the affective biasing of attention is not typically considered as a form of emotion regulation. 'Affect-biased attention' refers to selective attention processes [4] by which sensory systems are tuned to favor certain categories of affectively salient stimuli before they are encountered. In this opinion article, we draw on evidence from cognitive neuroscience and clinical and developmental psychology to argue that affect-biased attention is a form of emotion regulation. We argue that affectbiased attention, rather than being merely symptomatic of a reactive emotional response to a stimulus, is proactive in shaping perceptual experience. We further argue that habitual deployment of affect-biased attention has the potential to influence emotional responses to stressful events.

Although the theoretical literature on emotion regulation includes a number of sophisticated models [2,3], cognitive neuroscience approaches to emotion regulation have

Corresponding authors: Todd, R.M. (r.todd@acl.psych.toronto.edu); Thompson, E. (evan.thompson@utoronto.ca). been operationalized largely through studying two strategies: 'reappraisal', which involves cognitively re-evaluating an event to reduce its emotional impact, and 'suppression', which involves inhibiting the outward expression of emotion [5]. Although this research has been productive, influential models of emotion regulation have emphasized a wider range of processes with which emotional responses are continuously regulated, both before and after the occurrence of an emotional event, as well as below and above various thresholds of awareness [2,5]. For example, in the heat of the moment, when rapid responses are required, one may use more implicit and less cognitively taxing regulatory strategies, as recent research in implicit regulatory processes suggests [6-8]. Current emotion regulation models also include a number of additional regulatory strategies, such as voluntary distraction of attention, situation selection, and rumination (for a review, see [2,8]). In addition, developmental research has emphasized strategies of attentional shifting and focusing, as well as suppression of behavioral

\footnotetext{
Glossary

Affect-biased attention: attentional biases that give rise to preferential perception of a particular category of stimulus based on its relative affective salience.

Affective control setting: a habitual 'mental set,' based on our history of experience with what is motivationally relevant in a given context, which biases attention prior to an event.

Affective salience: the tendency of an item to stand out relative to its neighbors due to an association between its semantic meaning and emotional arousal. Attentional bias: a tendency to have one's attention initially drawn and sustained by one category of salient stimulus over another.

Attentional control settings: mental templates that bias top-down attention to specific object features in the service of specific short-term goals.

Bottom-up factors versus top-down factors: factors that reflect sensory stimulation by features of the environment, such as color, contrast, or sudden onset, versus cognitive factors that influence selective allocation of attention, such as knowledge, expectations, and current goals.

Emotional responses: physiological (cardiovascular, neuroendocrine) changes, actions, subjective feeling states, and changes in attentional focus associated with emotional arousal.

Exogenous spatial attention: salient external events serve as cues that shift or orient spatial attention in a reflexive manner.

Reactive control versus effortful control: forms of control that are relatively involuntary and reflexive, including approach and avoidance behaviors, versus voluntary, explicit, and flexible control over feelings and behavior, including the ability to inhibit a dominant response and activate a non-dominant response.

Reappraisal: cognitive reevaluation of a potentially emotionally arousing event in order to alter emotional impact.

Salience: the quality by which an aspect of the environment stands out relative to its surroundings.

Suppression: inhibition of behavior expressing emotional response.
} 
responses and delay of gratification (e.g., $[9,10])$. Yet, even sophisticated models do not include affect-biased attention within the taxonomy of regulatory processes. Rather, such attention is typically described as a symptom of reactive 'bottom-up' emotional response (e.g., [11,12]).

In this Opinion article we synthesize basic research on visual selective attention, clinical research on attentional biases in anxiety and depression, and research in affective science to support our proposal that affect-biased attention is a form of emotion regulation. This proposal is framed within the context of the 'Extended Model of Emotion Regulation', a comprehensive model that is grounded in developmental research and integrates models and findings from cognitive and affective neuroscience within a dynamic view of cognition/emotion processes (Box 1 and Figure 1). 'Affect-biased attention' refers to a pre-tuning of sensory systems so that certain categories of affectively salient stimuli are perceived over others. Here, we emphasize the regulatory role that affect-biased attention plays before the occurrence of an emotionally arousing event. For example, by biasing perception towards certain types of positive or negative stimuli, habitual affect-biased attention may modulate emotional responses to stressful events (see also [13]). Thus, if one's visual filters are pre-tuned to see more happy faces relative to angry faces in a crowd, one may be less likely to experience feelings of negative affect and heightened physiological arousal in a stressful situation than if one's attention is habitually biased towards negative expressions. Moreover, following classic work by Bruner [14], we argue that such habitual antecedent tuning of attention to context-specific and emotionally salient features of the environment is strongly shaped by development. In short, affect-biased attention consists in the implicit filtering processes, tuned over past experience, that modulate emotional responses to the world.

A recent definition of emotion regulation stipulates that a process is regulatory 'if and only if it is instantiated in pursuit of a goal to influence an ongoing or future emotion' [2]. Given that affect-biased attention is deployed habitually with the ongoing goal of modulating a habitually anticipated emotion (e.g., anxiety), it is regulatory in precisely this way. Nevertheless, from a dynamic perspective that emphasizes the ongoing unfolding of cognitive/emotional processes [15-17], 'emotion regulation' is not a process in its own right, but a useful heuristic, one that helps to delineate a taxonomy of cognition-emotion subcomponents [2]. Affect-biased attention is one of these subcomponents that modulate emotional responses at multiple temporal scales. When the full range of processes and their subcomponents is taken into consideration, however, it may prove difficult to define the boundary between emotion and regulation. For this reason, as several prominent emotion researchers have recently claimed [18,19], attempting to distinguish between emotion and its regulation may ultimately be impossible.

\section{Biased attention to affectively salient events}

Like emotion regulation, attentional selection is a broad category that includes a number of component processes. For example, attention can be deployed before or after an

\section{Box 1. Extended model of emotion regulation}

The extended model of emotion regulation (EMER) is a synthetic model, drawing on developmental psychology and cognitive neuroscience, which is contextualized within a dynamic view of cognition/emotion processes in the brain [15]. Drawing on Gross influential process model [2], we divide emotion regulation strategies into those that modulate emotional responses either before or after an emotionally salient event (Figure 1, x axis). However, distinct from dual-process models that draw a line between conscious and unconscious processes, or propose distinct neural pathways for each $[8,32]$, we draw on developmental research to incorporate the notion of multiple levels of control processes [47]. Drawing on Eisenberg's notion of levels of reactive and effortful control, we suggest that these range from reflexive strategies to more cognitively 'effortful' ones (Figure 1, y axis). These are based on graded levels of awareness/ availability for reflection, subserved by increasingly elaborated levels of processing $[3,70]$.

More effortful strategies become available with cognitive development, as levels of regulatory strategy become increasingly sophisticated, moving from limited stimulus-bound and temporally immediate responses to more deliberate and flexible strategies aimed at temporally distant goals [31]. To a certain extent, each leve of regulatory skill is thought to be dependent on development of the previous level. Specific regulatory strategies range from shifting attention away from a distressing object or event (e.g., [10]) and action tendencies to approach or withdraw, which emerge in infancy, to suppression of behaviors associated with approach, avoidance, or emotional display, which can be observed in toddlerhood [9]. Further skills, such as postponing immediate gratification for a more distant goal, emerge with cognitive milestones achieved in early childhood Others, such as reinterpretation or reappraisal of events in order to modify one's emotional response [1], rumination [8], and coping strategies such as problem-solving [71] rely on cognitive capacities that continue to mature into adolescence. The affect-biased attention we focus on here is one of a number of attentional deployment strategies, although it is one that is typically not discussed in terms of its regulatory role. Affect-biased attention may be sculpted gradually from infancy onward, although it may also be retrained by changing developmental or situational contexts.

Once available, more effortful and explicit strategies can be employed at a habitual level when primed in the laboratory or by a situational context, either prior or subsequent to an emotional event. For example, Mauss and colleagues [6] found that participants who were primed with words that evoked control rather than suppression of emotion were less likely to experience feelings of anger after anger induction. Williams and colleagues further found that priming participants with reappraisal goals reduced heart rate responses to an anxiety-inducing emotional challenge [7], suggesting that highorder strategies can be primed without reported awareness. However, we suggest that such high-order strategies as reappraisal may be possible only once the capacity for explicit re-appraisal is developmentally available.

According to the extended model, emotional interpretations involve the continuous unfolding of dynamical cognition-emotion interactions implicated in evaluation of events and regulation of responses to them at both psychological and neuronal levels [15]. In real time, over milliseconds to minutes, and in developmental time, over the course of years, increasingly effortful strategies draw on cycles of reprocessing of evaluative information into more complex hierarchical networks [72]. Information gleaned from initial rapid evaluations and habitual responses can be subsequently reprocessed at more reflective and effortful levels [72]. More reflective processing then feeds back to tune more reflexive perceptual filters and affective appraisals. Thus, according to this model, affect-biased attention, as a rapid, reflexive antecedent process of evaluation-based filtering, continuously shapes and is shaped by more reflective strategies within an emotional interpretation. 


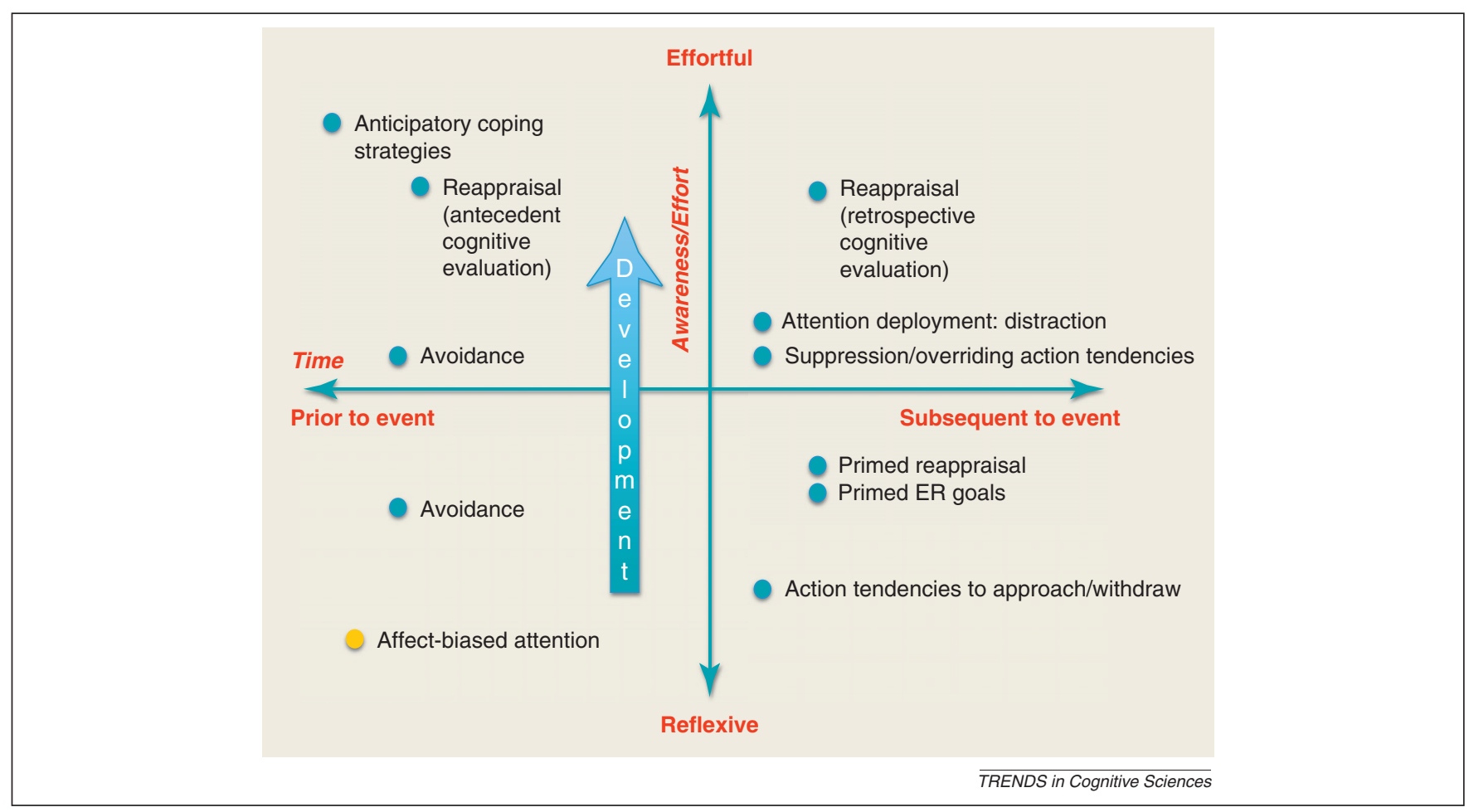

Figure 1. Extended model of emotion regulation. The $x$ axis represents time prior to and subsequent to an emotionally arousing event (time 0 ) and the $y$ axis represents the range of regulatory processes from habitual to effortful. Example regulatory processes are indicated with filled circles. Affect-biased attention, in yellow, is an antecedent and reflexive form of emotion regulation involving visual selective attention that tunes the contents of what we 'see' in the first place, before we encounter it.

event and can involve rapid or more extended processes, ranging from antecedent biasing of attention via control settings, to subsequent engagement of attention by stimulus salience, to later ease or difficulty of disengagement when an attentional shift is demanded [20]. Here, we discuss affect-biased attention as a specific form of selective attention that, unlike other attentional processes, has been typically discussed as a reactive symptom of emotion rather than a regulatory strategy [11].

Selective visual attention to some aspects of the environment at the expense of others is usually defined in terms of 'bottom-up' versus 'top-down' processes. Bottomup processes involve rapid and relatively reflexive attentional selection based on low-level visual features, intrinsic to the stimulus, such as color, contrast, and motion. Such aspects of 'objective salience' command attention because they are unexpected or stand out from the surrounding environment [21,22]. In contrast, effortful, top-down attention $[4,23]$ involves the establishment of 'attentional control settings' [24] - mental templates that hone attention to specific object features in the service of immediate goals, such as finding one's keys or successfully performing an experimental task. According to biased-competition models, top-down processes bias attention to relevant features of the environment before stimuli are presented and such pre-tuning of filters enhances the subsequent visual processing of those features. At the neural level, frontoparietal attention networks modulate visual cortex activation so that activity is enhanced in neurons or regions that are preferentially tuned to task-relevant stimuli, whereas activity is suppressed in neurons/regions sensitive to competing stimuli $[4,23]$.
Bottom-up objective salience is not the only kind of salience that can capture attention and thereby bias visual processing in a relatively reflexive manner. 'Affective salience' engages resources based on the emotional meaning of the stimulus. A robust body of emotion research has established that affectively salient stimuli, such as angry or smiling faces, or faces of loved ones are preferentially perceived and remembered when attentional resources are limited [25-28] and are more likely to attract overt attention when viewing complex scenes [29]. Although rapid behavioral responses and preferential amygdala activation have led many researchers in affective science to categorize them as reactive or bottom-up (e.g., [11]), we and others have proposed that affective salience mobilizes an alternative top-down system mediated by amygdala-centered networks $[30,31,32]$. We refer to it as a top-down system, because in top-down processes the features of the external environment that elicit attention are modulated by plans and goals. Such plans and goals can include affective and motivational goals [33], although these are not necessarily modulated by frontoparietal attentional networks.

According to our affective salience model (following [14]), affective salience networks tune the visual system so that it privileges incoming information based on one's history of experience of what is motivationally relevant in a given context, thereby generating a predisposition to attend to certain categories of stimuli over others. This predisposition functions as an 'affective control setting'. The result of an affective control setting is the attentional tuning that favors affectively salient stimuli, which we describe as affect-biased attention. Because affect-biased attention can be shaped habitually and reflexively, over 
time it can seem reactive or bottom-up-like. This view is consistent with appraisal models of emotion that stress that what is salient at any given time may be modulated by appraisals that are reflexive, habitual, and outside of focal attention [19]. These appraisals modulate emotional responses in the form of inclinations towards action [19]. According to our model of affect-biased attention, the goals that shape affect-biased attention are precisely such habitual appraisals of which one is typically unaware. The result of this kind of regulatory appraisal is affect-biased attentional tuning. Thus, we argue that biases for affective information reflect proactive application of affectively tuned top-down templates that are reflexively applied. It is important to note, however, that we are not suggesting that affect-biased attention to salient laboratory stimuli necessarily results in a full-blown physiological or experiential emotional response to the stimuli themselves; rather, we suggest that habits of selective attention revealed by laboratory tasks may influence physiological and experiential responses to more intense life stressors when they occur.

At the neural level, the claim that affective salience involves a form of top-down process is supported by findings that amygdala activation modulates visual cortex activation in a similar manner to visual cortex modulation by frontoparietal networks [28,34]. Thus, the amygdala is an important node in networks that support a form of biased attention that operates similarly to, but at times partially independent of, the biasing of attention associated with frontoparietal attention networks [35]. Because patterns of amygdala activation serve as well-documented neural markers of preferential responses to stimulus salience, our review of the empirical literature focuses on the amygdala; however, it is important to note that other regions, including the orbitofrontal cortex (OFC) and the pulvinar and inhibitory reticular nuclei of the thalamus, are also important hubs [35-37]. In conjunction with reciprocally connected regions of the amygdala, these regions also play key roles in integrating information from multiple cortical and subcortical regions to tag stimulus salience and direct attention to preferential features of the environment $[35,37]$.

Affect-biased attention may be subserved by 'arousalbiased competition', a mechanism similar to biased competition by top-down networks, in which regions of the visual cortex show enhanced activation for affectively salient items at the expense of relatively neutral ones [38]. Recent neuroanatomical research has given rise to neural models that implicate amygdala-thalamic connectivity in rapid orienting of attention to salient locations of a scene, thereby accounting for enhanced activation of regions tuned to salient stimuli and inhibition of distractors [35,39]. We suggest that biases in this system towards specific categories of stimuli underlie affectively biased competition. In contrast, more extended network interactions including the OFC are suggested to underlie other attentional processes, such as effortful attentional switching or disengagement [35]. However, there is also evidence that affective salience may bias attention without suppression of competing stimuli in a manner distinct from biased competition [40].

\section{Affective tuning can shift with age and context}

The affective salience model emphasizes that amygdala tuning, as a tractable index of motivational salience, is sensitive to both moment-to-moment and developmental context. Earlier notions of the amygdala's role in affective processing emphasized the idea that enhanced attention to threat, marked by enhanced amygdala activation, was 'hard-wired' in the service of evolutionary goals (e.g., [41]). A more recent conception is that, rather than being a hardwired 'automatic threat detector', the amygdala functions as a 'motivational relevance detector' $[33,42,43]$. Our own recent research suggests that amygdala activation indexing affect-biased attention may shift over the course of minutes with experimental context and over the course of years with developmental phase.

In a study examining the influence of evaluative goals on preferential amygdala activation to positive versus negative stimuli, Cunningham and colleagues [33] asked participants to evaluate the names of famous people. The results showed that the amygdala responded preferentially to names of celebrities that participants felt positive about when they were asked to evaluate positivity and to names of celebrities they felt negative about when asked to rate negativity (Figure 2c,d). Thus, the response pattern of the amygdala shifted as the affective salience of stimuli changed according to the moment-to-moment goals of the perceiver. We argue that relative affective salience was pre-tuned by task context, allowing the establishment of affect-biased attention to the stimuli that was dependent on the task at hand. Such contextual tuning of affective salience is equivalent to contextual modulation of attention by control settings, consistent with our claim that affect-biased attention functions as a control setting tuned by affective salience. Because of amygdala links to brainstem and hypothalamus regions that modulate subsequent physiological responses associated with emotional arousal, such pre-tuning has the potential to regulate subsequent emotional responses - even though preferential amygdala activation to a stimulus does not necessarily result in a direct full fledged emotional response.

In another series of studies investigating the role of developmental phase in amygdala response to affective salience, we showed groups of young children (4-6 years and 6-9 years) and young adults (18-35 years) images of their mother and an appearance-matched stranger showing happy and angry/displeased expressions [44]. When freely viewing the faces, children, but not young adults, showed greater amygdala activation in response to happy versus angry faces (Figure $2 \mathrm{a}$ and $\mathrm{b}$ ). This finding suggests that, in contrast to adults, young children may find smiling expressions - considered to be rewarding in their own right - to be more salient than angry ones. This interpretation is consistent with a well-documented positivity bias in older adults [45], accompanied by preferential amygdala activation for positive versus negative stimuli [46]. Thus, the relative salience of positive and negative facial expressions may be different at different developmental stages, suggesting normative developmental changes in affect-biased attention, or what individuals are predisposed to attend to, with both young children and older adults showing evidence of a positivity bias relative to young adults. This 
(a)

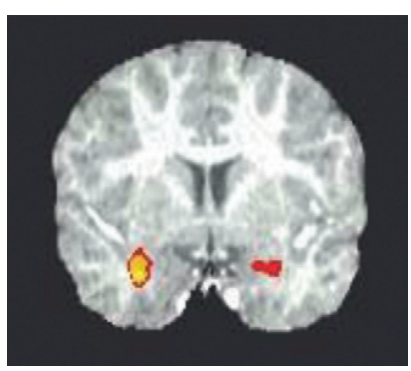

(b)

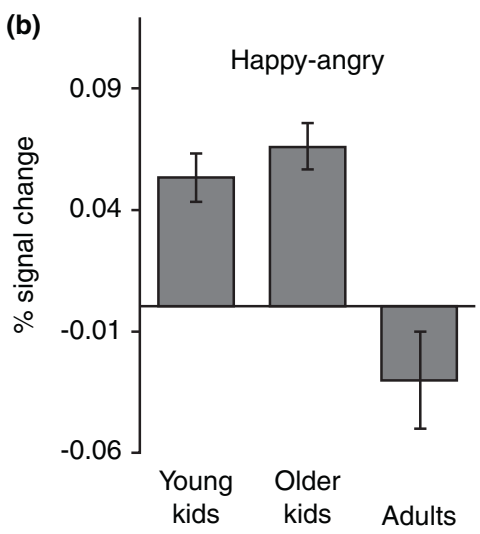

(c)

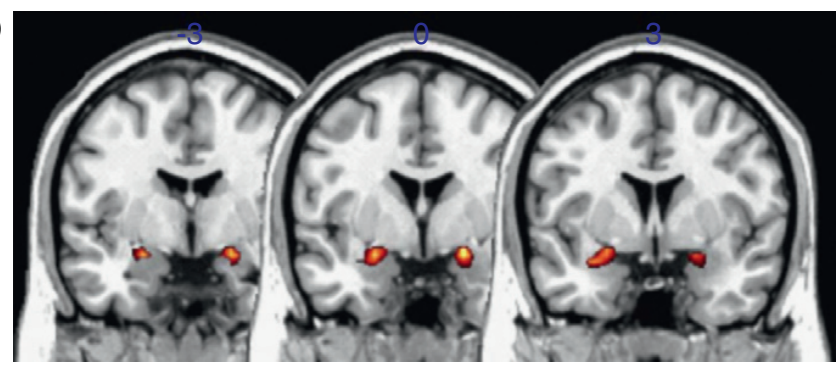

(d)

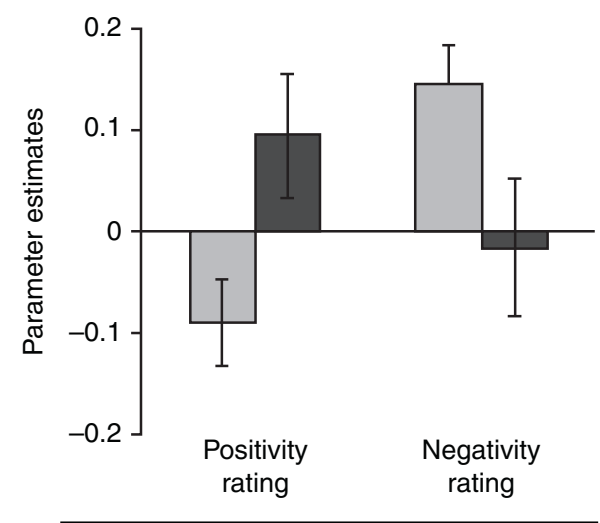

Key: $\square$ Negative condition $\square$ Positive condition

TRENDS in Cognitive Sciences

Figure 2. Preferential amygdala activation to positive versus negative stimuli changes with developmental and experimental context. (a) Activation map showing greater activation for happy than angry faces in young children. Reproduced, with permission, from [44] from Oxford University Press. (b) Difference between bilateral amygdala activation for happy - angry in 3-6 year old children, 6-9 year old children, and young adults. Data from [44]. (c) Amygdala regions showing a valence by behavioural condition effect showing greater amygdala for positive stimuli during positive evaluations and for negative stimuli for negative evaluations. Reproduced, with permission, from [33] from Sage Publications. (d) Beta weights associated with bilateral amygdala activation by participant ratings of positivity and negativity. Reproduced, with permission, from [33].

pattern does not necessarily illustrate age-related differences in the degree of affective bias or for the capacity for suppression of biases, but simply a change in what is more motivationally important at a given stage of life. However, it is important to note that, beyond normative developmental trends, individual differences in specific biases may become more entrenched with age [47].

Affect-biased attention may also influence downstream regulatory processes - which in turn may show parallel agerelated changes. Some emotional facial expressions are thought to elicit overlearned action tendencies either to act or refrain from acting, or to approach or withdraw $[48,49]$ - forms of reflexive post-stimulus control. In young adults, a region of lateral OFC has been found to be active when a required response (e.g., withdraw) is incongruent with the action tendency signaled by a previously presented face (e.g., smiling). Deactivation of this region impairs performance during incongruent trials [50], suggesting that lateral OFC plays a key role in linking stimulus salience with the need to override habitual actions using explicit control processes. In a second study, we examined fMRI activation in children and young adults when the presentation of a happy or angry face (mothers and matched strangers) preceded a cue either to press a button or withhold an action [51]. In this study, activation in lateral OFC decreased with age group for the difference between angry and happy faces, showing a parallel pattern to that showed by the amygdala in the free viewing task - but only during incongruent trials when an action had to be withheld. These findings indicate that not only does amygdala activation linked to the affective salience of a stimulus differ across age groups, but the way affective salience interacts with activation linked to post-event regulatory processes differs as well. In this case, affect-biased attention favoring a given category of stimulus may influence overlearned habitual responses following an event, which in turn influence explicit control required to override the habitual response. Thus, not only may the tuning of affective biases change across the lifespan, but such tuning can serve as a form of attentional regulation that can influence behavior via a cascade of regulatory processes along the habitual versus effortful and pre- versus post-event axes. However, future research is required to directly link brain activation patterns reported here with pre-tuned biases for positive stimuli in young children.

\section{Individual differences in affect-biased attention}

We suggest that well-documented individual differences in affect-biased attention to positive or negative stimuli reflect individual differences in habitual employment of affectively motivated control settings, which play a regulatory role by influencing subsequent regulatory processes. A recent study [52] found that participants who were high in a form of neuroticism linked to volatility, which involves predisposition towards anger or irritation, as well as affectbiased attention for negative images, showed greatest amygdala activation for negative images, regardless of 
whether they had to approach or avoid them. In contrast, participants who were high in neuroticism-avoidance, which is characterized by a general pattern of avoidance that is not linked to attentional biases towards a particular valence or category, showed greater amygdala activation when they had to approach a stimulus regardless of valence. These findings suggest that habitual individual differences in affect-biased attention influence the salience of willfully performed actions, which in turn play a role in post-event explicit regulatory processes. In this case, too, temporally subsequent and more explicit regulatory processes are built on more reflexive ones.

\section{Attentional biases influence emotional responding and are trainable}

It is well established that some people are predisposed to focus on threatening aspects of a scene, whereas others focus on the positive aspects [53,54]. For example, imagine looking out at a sea of faces before giving a lecture. Some people habitually zoom in on frowning or bored faces. Others are predisposed to find smiles of approval. A robust body of research has linked attentional biases toward threatening versus neutral or positive images with normative and clinical anxiety [53], and attentional biases for rewarding stimuli have been shown to be associated with such personality traits as extraversion [54].

Studies investigating attentional biases to stimulus category typically use exogenous spatial cueing tasks to measure bias, and most often employ variations of a dot probe task. In a typical dot probe task, response times to a probe in a spatial location previously occupied by a stimulus of one valence (e.g., an angry face) are compared with those to a probe in a location previously occupied by a stimulus of another valence (e.g., a smiling or neutral face) [55]. Differences in response time are used as a measure of biases involving both initial attentional capture by and subsequent difficulty to disengage attention from the former location of particular valence or category of stimulus [55]. We suggest that such attentional capture by specific stimulus categories may reflect affectively biased control settings. Moreover, research using dot probe tasks suggests that habitual attentional biases predict emotional responsiveness to stressors, suggesting that they modulate emotional responding [56,57]. For example, biases for implicitly presented negative stimuli have been positively associated with cortisol levels both in response to an inlaboratory stressor (having to give a five minute talk) four months later, as well to a major final exam eight months later [58]. Although this does not provide direct evidence that attentional biases modulate emotional responses, an important body of research on cognitive bias modification has provided promising evidence that experimental manipulation of attentional biases can influence subsequent anxiety symptoms and responses to stressors.

Attention bias modification (ABM) is a form of cognitive behavior modification that uses an exogenous cueing task to manipulate biases favoring the valence or specific category of a stimulus [59]. In ABM, participants repeatedly perform a dot probe task that manipulates the frequency of presentation of probes in the place of threatening relative to neutral stimuli. Presenting probes in the place of neutral stimuli more frequently than threatening ones reduces biases to threat in a manner that is implicit and based on repeated practice. A meta-analysis finding a consistent effect of ABM in shifting attentional biases and reducing anxiety scores in both clinical and non-clinical populations [54] suggests that negative attentional biases render adults more vulnerable to anxiety symptoms and responses to stress, and that manipulation of biases effectively reduces anxiety. However, it should be noted that effect sizes for ABM influence on anxiety are small and do not always replicate reliably [60].

\section{Attentional biases emerge early, influence behavioral outcomes, and are trainable in children}

Attentional biases, which are associated with common genetic variations [61], can be measured as early in development as five years [62]. They are also associated with behavioral outcomes early in development. For example, a recent study demonstrated that attentional biases to threat predicted whether temperamentally inhibited children would demonstrate social withdrawal behaviors at age five [63]. Moreover, recent research has extended ABM training to anxiety reduction in children, as well. In a recent randomized control trial, clinically anxious children who demonstrated attention biases for threat were either assigned to an ABM condition or one of two control conditions [64]. After four training sessions over four weeks, only the children in the ABM condition showed reduced attentional biases and anxiety symptoms as measured in both clinician interviews and on a DSM-IV anxiety severity scale measured in a post-treatment session. These findings demonstrate that tuning of habitual deployment of affective control sets over an extended period of time can modulate patterns of emotional response in children, consistent with an important role for affect-biased attention in regulating emotional responses to subsequent stressful events. Longterm effects of such interventions in children - particularly in comparison with adults - will be an important area for future research.

\section{Direct measures of affect-biased attention}

It is important to note that exogenous spatial cueing tasks, such as the dot probe, do not directly measure/manipulate antecedent tuning of perceptual filters to specific categories of stimuli. Thus, they are not direct measures of affectbiased attention as a form of habitual control setting that precedes stimulus presentation. Rather, they measure rapid capture and/or sustained retention of exogenous spatial attention following presentation of salient stimuli [55]. Thus, they measure the propensity to be oriented by affectively salient stimuli to the spatial location at which they occur, rather than an individual's pre-tuning to specific categories of stimulus; however, they may reflect such pre-tuning. Although less commonly used for ABM, visual search tasks that require participants to locate a specific facial expression among an array of distractors are better measures of the kind of pre-stimulus filtering we describe as affect-biased attention. Here, salience-based biases are measured as differences in response times when locating search targets of different stimulus categories. To date, at least one series of studies has trained attentional bias by 
using smiling faces as targets among an array of faces with negative expressions and comparing it to a control condition with flower targets [65]. Repeated training reduced reported stress in response to a final exam in students and reduced stress responses and cortisol in telemarketers. Although replication is required, this study offers more direct evidence that training habits of affect-biased attention over time regulates aspects of emotional response to subsequent stressors.

\section{Future directions}

Despite the fact that there are suggestive data supporting our proposal that affect-biased attention is a form of emotion regulation, further research is required to test directly the influence of affect-biased attention on behavioral and physiological measures associated with emotional response to emotional challenges, as well as on measures of specific downstream regulatory processes (Box 2). Future research can also draw on animal models and human neurogenetics to elucidate the role of serotonin, as well as other neuromodulators, such as dopamine and norepinephrine, in affect-biased attention. In addition, whereas a number of computational models of bottom-up visual salience predict attention deployment based on low-level visual features (e.g., [21]) and even incorporate aspects of semantic meaning [66], future models can incorporate affective salience parameters to predict attention deployment.

We have suggested that affect-biased attention is tuned through experience over development, a claim supported by research investigating the role of early experience in sensitivity to specific expressions [67]. Associative learning has been proposed as one mechanism underlying such developmental tuning [67], but future research is required to test this hypothesis directly. Finally, given recent interest in meditation techniques for training attentional processes associated with emotion regulation [68,69], another important area for future research is investigation of these techniques for training affective control sets and their influence on subsequent emotional responding.

\section{Box 2. Questions for future research}

- Does affective pre-tuning towards stimulus categories, as can be measured by visual search tasks for example, directly influence downstream emotional (e.g., physiological, self-report) responses? If so, by what mechanisms?

- Can attentional biases be predicted by generating comprehensive computational models of stimulus salience that account for affective salience?

- What learning processes underlie the tuning of affective biases over development?

- Given links between serotonin transporter gene variations and attentional biases, what specifically is the role of serotonin in tuning affect-biased attention and what influence does training have on serotonin-mediated processes? Do other neuromodulators, such as noradrenaline and dopamine, also play a role? Convergent data from animal models and neurogenetic human research can help to answer these questions.

- What meditative attention regulation techniques are effective in modulating affective control settings and how do they influence patterns of emotional response?

\section{Concluding remarks}

If affect-biased attention is a form of emotion regulation, as we have argued here, one might wonder whether any form of emotion is unregulated - and hence whether the term 'emotion regulation' is ultimately meaningful. In the end, when one delineates the full range of subcomponents that modulate cognition/emotion interactions over multiple timescales, one may conclude that there is at no stage a 'pure' emotional response that is not always already regulated in some way. Not only may it be impossible to distinguish 'emotional' from 'cognitive' processes in the brain $[15,16]$, it may be extremely rare to find pure emotional reactivity unregulated by some form of attentional process. Nonetheless, we suggest that an inclusive view of emotion regulation continues to serve as a useful heuristic for understanding behavioral and attentional processes that modulate emotion.

\section{References}

1 Gross, J.J. (2001) Emotion regulation in adulthood: timing is everything. Curr. Dir. Psychol. Sci. 10, 214-219

2 Gross, J.J. et al. (2011) Cognition and Emotion Lecture at the 2010 SPSP Emotion Preconference. Cogn. Emot. 25, 765-781

3 Eisenberg, N. et al. (2010) Emotion-related self-regulation and its relation to children's maladjustment. Annu. Rev. Clin. Psychol. 6, $495-525$

4 Corbetta, M. and Shulman, G.L. (2002) Control of goal-directed and stimulus-driven attention in the brain. Nat. Rev. Neurosci. 3, 201-215

5 Gross, J.J. and Thompson, R.A. (2007) Emotion regulation: conceptual foundations. In Handbook of Emotion Regulation (Gross, J.J., ed.), pp. 3-26, Guilford Press

6 Mauss, I.B. (2007) Automatic emotion regulation during anger provocation. J. Exp. Soc. Psychol. 43, 698-711

7 Williams, L.E. et al. (2009) The unconscious regulation of emotion nonconscious reappraisal goals modulate emotional reactivity. Emotion 9, 847-854

8 Gyurak, A. et al. (2011) Explicit and implicit emotion regulation: a dual-process framework. Cogn. Emot. 25, 400-412

9 Fox, N.A. and Calkins, S.D. (2003) The development of self-control of emotion: intrinsic and extrinsic influences. Motiv. Emot. 27, 7-16

10 Rothbart, M.K. and Derryberry, D. (1981) Development of individual differences in temperament. In Advances in Developmental Psychology (Lamb, M.E. and Brown, A.L., eds), pp. 37-86, Erlbaum

11 Soares, S.C. et al. (2009) Some animal specific fears are more specific than others: Evidence from attention and emotion measures. Behav. Res. Ther. 47, 1032-1042

12 Silvers, J.A., et al. Age-related differences in emotional reactivity, regulation, and rejection sensitivity in adolescence. Emotion 12, (in press)

13 Wadlinger, H.A. and Isaacowitz, D.M. (2011) Fixing our focus: training attention to regulate emotion. Pers. Soc. Psychol. Rev. 15, 75-102

14 Bruner, J.S. and Goodman, C.C. (1947) Value and need as organizing factors in perception. Journal. Abnorm. Psychol. 42, 33-44

15 Lewis, M.D. (2005) Bridging emotion theory and neurobiology through dynamic systems modeling. Behav. Brain Sci. 28, 169-194 discussion 194-245

16 Pessoa, L. (2008) On the relationship between emotion and cognition. Nat. Rev. Neurosci. 9, 148-158

17 Scherer, K.R. (2009) Emotions are emergent processes: they require a dynamic computational architecture. Philos. Trans. R. Soc. Lond. B: Biol. Sci. 364, 3459-3474

18 Kappas, A. (2011) Emotion is not just an alarm bell - it's the whole tootin' fire truck. Cogn. Emot. 25, 785-788 discussion 789-793

19 Mesquita, B. and Frijda, N.H. (2011) An emotion perspective on emotion regulation. Cogn. Emot. 25, 782-784 discussion 789-793

20 Cosmelli, D. (2009) Attending to the stream of consciousness: A methodological challenge. In From Attention to Goal Directed Behavior (Aboitiz, F. and Cosmelli, D., eds), pp. 83-103, Springer-Verlag

21 Itti, L. et al. (1998) A model of saliency-based visual attention for rapid scene analysis. IEEE Trans. Pattern Anal. Mach. Intell. 20, 1254-1259 
22 Hou, X. and Zhang, L. (2007) Saliency detection: a spectral residual approach. Proc. IEEE CVPR 2007, 1-7

23 Desimone, R. and Duncan, J. (1995) Neural mechanisms of selective visual attention. Annu. Rev. Neurosci. 18, 193-222

24 Folk, C.L. et al. (1992) Involuntary covert orienting is contingent on attentional control settings. J. Exp. Psychol. Hum. Percept. Perform. 18, 1030-1044

25 Anderson, A.K. (2005) Affective influences on the attentional dynamics supporting awareness. J. Exp. Psychol. Gen. 134, 258-281

26 Anderson, A.K. and Phelps, E.A. (2001) Lesions of the human amygdala impair enhanced perception of emotionally salient events. Nature 411, 305-309

27 De Martino, B. et al. (2009) Enhanced processing of threat stimuli under limited attentional resources. Cereb. Cortex 19, 127-133

28 Lim, S.L. et al. (2009) Segregating the significant from the mundane on a moment-to-moment basis via direct and indirect amygdala contributions. Proc. Natl. Acad. Sci. U.S.A. 106, 16841-16846

29 Humphrey, K. et al. (2012) Salience of the lambs: A test of the saliency map hypothesis with pictures of emotive objects. J. Vis. 12, http:// dx.doi.org/10.1167/12.1.22

30 Todd, R.M. and Anderson, A.K. Salience, state, and expression: the influence of specific aspects of emotion on attention and pereption. In: Handbook of Cognitive Neuroscience (Kosslyn, K.O.a.S.M., ed.), Oxford University Press (in press)

31 Cunningham, W.A. and Brosch, T. (2012) Motivational salience: amygdala tuning from traits, needs, values and goals. Curr. Dir. Psychol. Sci. 21, 54-59

32 Vuilleumier, P. (2005) How brains beware: neural mechanisms of emotional attention. Trends Cogn. Sci. 9, 585-594

33 Cunningham, W.A. et al. (2008) Affective flexibility: evaluative processing goals shape amygdala activity. Psychol. Sci. 19, 152-160

34 Pessoa, L. et al. (2002) Attentional control of the processing of neural and emotional stimuli. Brain Res. Cogn. Brain Res. 15, 31-45

35 Zikopoulos, B. and Barbas, H. (2012) Pathways for emotions and attention converge on the thalamic reticular nucleus in primates. $J$. Neurosci. 32, 5338-5350

36 Barrett, L.F. and Bar, M. (2009) See it with feeling: affective predictions during object perception. Philos. Trans. R. Soc. Lond. B: Biol. Sci. 364, 1325-1334

37 Pessoa, L. and Adolphs, R. (2010) Emotion processing and the amygdala: from a 'low road' to 'many roads' of evaluating biological significance. Nat. Rev. Neurosci. 11, 773-783

38 Mather, M. and Sutherland, M.R. (2011) Arousal-biased competition in perception and memory. Perspect. Psychol. Sci. 6, 114-133

39 Pessoa, L. (2010) Emergent processes in cognitive-emotional interactions. Dialogues Clin. Neurosci. 12, 433-448

40 Wieser, M.J. et al. (2011) Sustained preferential processing of social threat cues: bias without competition? J. Cogn. Neurosci. 23, 1973-1986

$41 \mathrm{Ohman}$, A. et al. (2007) On the unconscious subcortical origin of human fear. Physiol. Behav. 92, 180-185

42 Todd, R.M. and Anderson, A.K. (2009) Six degrees of separation: the amygdala regulates social behavior and perception. Nat. Neurosci. 12, 1217-1218

43 Adolphs, R. et al. (2005) A mechanism for impaired fear recognition after amygdala damage. Nature 433, 68-72

44 Todd, R.M. et al. (2011) The changing face of emotion: age-related patterns of amygdala activation to salient faces. Soc. Cogn. Affect. Neurosci. 6, 12-23

45 Mather, M. and Carstensen, L.L. (2003) Aging and attentional biases for emotional faces. Psychol. Sci. 14, 409-415

46 Mather, M. et al. (2004) Amygdala responses to emotionally valenced stimuli in older and younger adults. Psychol. Sci. 15, 259-263

47 Lewis, M.D. and Todd, R.M. (2007) The self-regulating brain: corticalsubcortical feedback and the development of intelligent action. Cogn. Dev. 22, 406-430
48 Roelofs, K. et al. (2009) On the neural control of social emotional behavior. Soc. Cogn. Affect. Neurosci. 4, 50-58

49 Blair, R.J. et al. (1999) Dissociable neural responses to facial expressions of sadness and anger. Brain 122, 883-893

50 Volman, I. et al. (2011) Anterior prefrontal cortex inhibition impairs control over social emotional actions. Curr. Biol. 21, 1766-1770

51 Todd, R.M. et al. (2012) Witholding response in the face of a smile: agerelated differences in prefrontal sensitivity to Nogo cues following happy and angry faces. Dev. Cogn. Neurosci. 2, 340-350

52 Cunningham, W.A. et al. (2010) Aspects of neuroticism and the amygdala: chronic tuning from motivational styles. Neuropsychologia 48, 3399-3404

53 Bar-Haim, Y. et al. (2007) Threat-related attentional bias in anxious and nonanxious individuals: a meta-analytic study. Psychol. Bull. 133, $1-24$

54 Hakamata, Y. et al. (2010) Attention bias modification treatment: a meta-analysis toward the establishment of novel treatment for anxiety. Biol. Psychiatry 68, 982-990

55 Fox, E. et al. (2002) Attentional bias for threat: evidence for delayed disengagement from emotional faces. Cogn. Emot. 16, 355-379

56 MacLeod, C. et al. (2009) Whither cognitive bias modification research? Commentary on the special section articles. J. Abnorm. Psychol. 118 , 89-99

57 Hertel, P.T. and Matthews, A. (2011) Cognitive bias modification: past perspectives, current findings, and future applications. Perspect. Psychol. Sci. 6, 521-536

58 Fox, E. et al. (2010) Preconscious processing biases predict emotional reactivity to stress. Biol. Psychiatry 67, 371-377

59 Hertel, P.T. et al. (2011) Recollection is impaired by the modification of interpretation bias. J. Abnorm. Psychol. 120, 902-910

60 Hallion, L.S. and Ruscio, A.M. (2011) A meta-analysis of the effect of cognitive bias modification on anxiety and depression. Psychol. Bull. 137, 940-958

61 Pergamin-Hight, L. et al. (2011) Variations in the promoter region of the serotonin transporter gene and biased attention for emotional information: a meta-analysis. Biol. Psychiatry 71, 371-378

62 Kujawa, A.J. et al. (2011) Attentional biases for emotional faces in young children of mothers with chronic or recurrent depression. $J$. Abnorm. Child Psychol. 39, 125-135

63 Perez-Edgar, K. et al. (2011) Attention biases to threat link behavioral inhibition to social withdrawal over time in very young children. $J$. Abnorm. Child Psychol. 39, 885-895

64 Eldar, S. et al. (2012) Attention bias modification treatment for pediatric anxiety disorders: a randomized controlled trial. Am. J. Psychiatry 169, 213-220

65 Dandeneau, S.D. et al. (2007) Cutting stress off at the pass: reducing vigilance and responsiveness to social threat by manipulating attention. J. Pers. Soc. Psychol. 93, 651-666

66 Cerf, M. et al. (2009) Faces and text attract gaze independent of the task: experimental data and computer model. J. Vis. 9, http:// dx.doi.org/10.1167/9.12.10

67 Pollak, S.D. and Tolley-Schell, S.A. (2003) Selective attention to facial emotion in physically abused children. J. Abnorm. Psychol. 112, 323-338

68 Farb, N.A. et al. (2010) Minding one's emotions: mindfulness training alters the neural expression of sadness. Emotion 10, 25-33

69 Lutz, A. et al. (2008) Attention regulation and monitoring in meditation. Trends Cogn. Sci. 12, 163-169

70 Zelazo, P.D. (2004) The development of conscious control in childhood. Trends Cogn. Sci. 8, 12-17

71 Saarni, C. (1997) Coping with aversive feelings. Motiv. Emot. 21, $45-63$

72 Cunningham, W.A. and Zelazo, P.D. (2007) Attitudes and evaluations: a social cognitive neuroscience perspective. Trends Cogn. Sci. 11, 97-104 